\title{
Highly tunable method to generate sinc-shaped Nyquist pulses from a rectangular frequency comb
}

\author{
Marcelo A. Soto ${ }^{1, *}$, Mehdi Alem ${ }^{1}$, Mohammad Amin Shoaie ${ }^{2}$, Armand Vedadi' ${ }^{2}$, Camille-Sophie Brès ${ }^{2}$, \\ Luc Thévenaz ${ }^{1}$, and Thomas Schneider ${ }^{3}$ \\ ${ }^{1}$ EPFL Swiss Federal Institute of Technology, Group for Fibre Optics (GFO), SCI-STI-LT, Station 11, CH-1015 Lausanne, Switzerland \\ ${ }^{2}$ EPFL Swiss Federal Institute of Technology, Photonic Systems Laboratory (PHOSL), SCI-IEL, Station 11, CH-1015 Lausanne, Switzerland \\ ${ }^{3}$ Institut für Hochfrequenztechnik, Hochschule für Telekommunikation Leipzig, Gustav-Freytag-Str. 43-45, 04277 Leipzig, Germany \\ *Email: marcelo.soto@epfl.ch
}

\begin{abstract}
A method to produce highly-stable optical sinc-shaped pulses is proposed based on the generation of a rectangular-spectrum frequency comb. Nyquist pulses with $<1 \%$ power distortion, 82-fs jitter and more than $40 \mathrm{~dB}$ SNR are achieved.

OCIS codes: (060.4510) Optical communications; (060.4080) Modulation; (320.5550) Pulses.
\end{abstract}

\section{Introduction}

The demand for higher capacity optical fiber networks is on the rise. Limitations of viable alternatives to expand the bandwidth of optical telecommunications beyond the constraints imposed by erbium-doped fiber amplifiers (EDFAs) has funneled recent research efforts towards increasing the spectral efficiency of transmitted signals. Besides multiplying the number of transmitted bits per symbol with multi-level phase and/or amplitude modulation schemes in coherent transmission systems, efforts are underway to contain the transmitted signal spectrum within a band-width that is as close as possible to the symbol rate, which is the lower bound dictated by the Nyquist theorem [1]. Such a trend is observed in recent demonstrations of Nyquist-WDM transmissions [2], where the baseband signal is digitally processed so as to achieve a rectangular spectrum. An all-optical counterpart, in which the optical symbol pulse transporting data has a sinc temporal shape and a rectangular spectrum, would pave the way for the growth of the data throughput by efficiently aggregating data in both time and frequency domains. In addition, for applications such as all-optical sampling or optical analog-to-digital conversion (ADC), the possibility to use optical Nyquist-limited pulses for data sampling could ease bandwidth constraints and improve performances [3].

All-optical techniques to generate Nyquist pulses that have been reported either use parametric amplification [4] or a spatial light modulator together with mode-locked lasers [5]. Besides the high complexity of the proposed methods, these techniques fail to generate ideal sinc pulses, leading to non-rectangular spectrum and hence to inefficient use of the spectral bandwidth.

In this paper, a method to produce perfect optical Nyquist pulses is proposed based on the generation of a phaselocked frequency comb with an ideal rectangular spectral shape. We demonstrate the effectiveness and flexibility of the method using two cascaded electro-optic intensity modulators (EOMs), achieving sinc-shaped Nyquist pulses with variable width down to $8.9 \mathrm{ps}$, low jitter and high signal-to-noise ratio (SNR $>40 \mathrm{~dB}$ ).

\section{Theory and principle of the proposed method}

The method proposed in this paper is based on the direct generation of a flat frequency comb containing equallyspaced and phase-locked spectral lines. This comb corresponds to a train of sinc pulses in the time domain. While the pulse period is given by the frequency spacing between adjacent lines, the pulse duration is defined by the bandwidth of the frequency comb. If every spectral component of a flat frequency comb (with $N$ lines, frequency spacing $\Delta f$ and similar phase $\phi$ around the carrier frequency $\left.f_{0}\right)$ is represented by $\exp \left(2 i \pi\left(f_{0}+n \Delta f\right) t+i \phi\right)$, for $n=-(N-1) / 2, \ldots,(N-1) / 2$, the field of such a comb can be expressed in the time domain as:

$$
E(t)=\sum_{n=-\frac{N-1}{2}}^{\frac{N-1}{2}} e^{2 i \pi\left(f_{0}+n \Delta f\right) t+i \phi}=e^{2 i \pi f_{0} t+i \phi} \sum_{n=-\frac{N-1}{2}}^{\frac{N-1}{2}} e^{2 i \pi n \Delta f t}=\frac{\sin (\pi N \Delta f t)}{\sin (\pi \Delta f t)} e^{2 i \pi f_{0} t+i \phi}
$$

The intensity of the generated pulses can be represented by $I(t)=|E(t)|^{2}=\sin ^{2}(\pi N \Delta f t) / \sin ^{2}(\pi \Delta f t)$, while the period of pulse train is $T=1 / \Delta f$ and the zero-crossing pulse duration is $\tau=2 T / N=2 / N \Delta f$.

The method here proposed is based on the possibility to generate 2 or 3 spectral lines, with high suppression of higher-order sidebands, using electro-optic intensity modulators [6]. This way, a frequency comb can be produced by simply cascading EOMs with appropriate bias and RF voltages. Depending on the number of frequency lines that each EOM generates, it is possible to have an odd or even number of spectral lines in the generated comb. For instance, if two EOMs are used, one driven at a frequency $f$ and the other at $3 f$ without any carrier suppression, a 
comb with 9 spectral lines and frequency spacing $f$ covering the optical bandwidth of $9 f$ can be generated. In particular, the output field of an intensity EOM with normalized bias voltage $\epsilon=\frac{V_{B}}{V_{\pi}}$, and RF signal $\alpha=\frac{v_{S}}{V_{\pi}}$ is [6]:

$$
E(t)=\sum_{k=-\infty}^{+\infty}(-1)^{k}\left(\cos (\pi \epsilon) J_{2 k}(\pi \alpha) \cos \left(\omega_{0} t+2 k \omega_{s} t\right)+\sin (\pi \epsilon) J_{2 k-1}(\pi \alpha) \cos \left(\omega_{0} t+(2 k-1) \omega_{s} t\right)\right)
$$

where $V_{\pi}$ is the half-wave voltage of the EOM and $J_{k}$ is the Bessel function of the first kind. In order to achieve a flat frequency comb, it is necessary that every EOM produces lines with the same amplitude. To achieve this condition, the bias voltage $V_{B}$ and the modulating RF signal $v_{S}$ applied to every EOM must satisfy the following equation:

$$
V_{B}=\frac{V_{\pi}}{\pi} \tan ^{-1}\left\{-J_{0}\left(\pi \frac{v_{s}}{V_{\pi}}\right) / J_{1}\left(\pi \frac{v_{s}}{V_{\pi}}\right)\right\}
$$

On the other hand, in order to produce a rectangular-shaped comb, the higher-order modulation components have to be strongly suppressed. This can be achieved by adjusting the RF signal. As shown in Fig. 1, a suppression of more than $20 \mathrm{~dB}$ (with respect to the carrier and first-order sidebands) can be achieved for the second (and higher) order modulations by decreasing the modulating signal below $0.25 V_{\pi}$.

\section{Experimental setup}

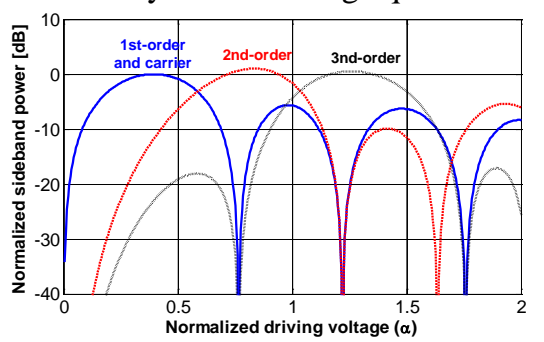

Fig. 1. Power of the lower-order sidebands vs the normalized RF voltage (equalized case)

Fig. 2 shows the experimental setup, in which the light from an external cavity laser (ECL) at $1550 \mathrm{~nm}$ is launched into two cascaded Mach-Zehnder modulators (MZMs). Note that, the microwave generators have been synchronized using a common time base, while the relative phase among them has been carefully adjusted, leading in this way to almost-ideal and symmetric sinc-shaped Nyquist pulses. A semiconductor optical amplifier (SOA) and an EDFA have been used, together with two tunable optical filters (TOF), to measure the pulses on a $500-\mathrm{GHz}$ optical sampling oscilloscope, which requires a minimum average power of $0 \mathrm{dBm}$ for reliable jitter and SNR measurements. Note that the optical filters are used only to reduce amplified spontaneous emission (ASE) noise generated in the optical amplifiers and are not required for spectral shaping as typically employed in other methods generating Nyquist pulses. An optical spectrum analyzer (OSA) with a resolution bandwidth of $0.01 \mathrm{~nm}$ is used to measure the spectrum of the generated phase-locked, rectangular-shaped frequency combs.

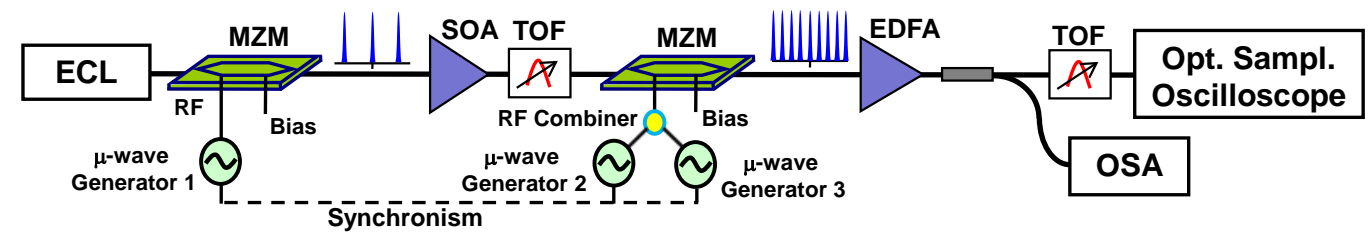

Fig. 2. Experimental setup for ideal and higly-stable Nyquist pulse generation based on a rectangular frequency comb.

\section{Experimental results and discussion}

In order to verify the flexibility of the proposed method, frequency combs with different bandwidth and number of spectral components have been generated. First, modulating the first and second EOM with RF signals at $30 \mathrm{GHz}$ and $10 \mathrm{GHz}$, respectively, a comb with $N=9$ spectral components separated by $\Delta f=10 \mathrm{GHz}$, and expanding over a bandwidth of $90 \mathrm{GHz}$, has been generated, as shown in Fig. 3(a). The spectral separation $\Delta f$ and the bandwidth of the comb can be easily modified by changing the frequency of the modulating signals. Furthermore, in order to increase the number of lines of the comb, the second EOM has been driven by two RF signals combined in the
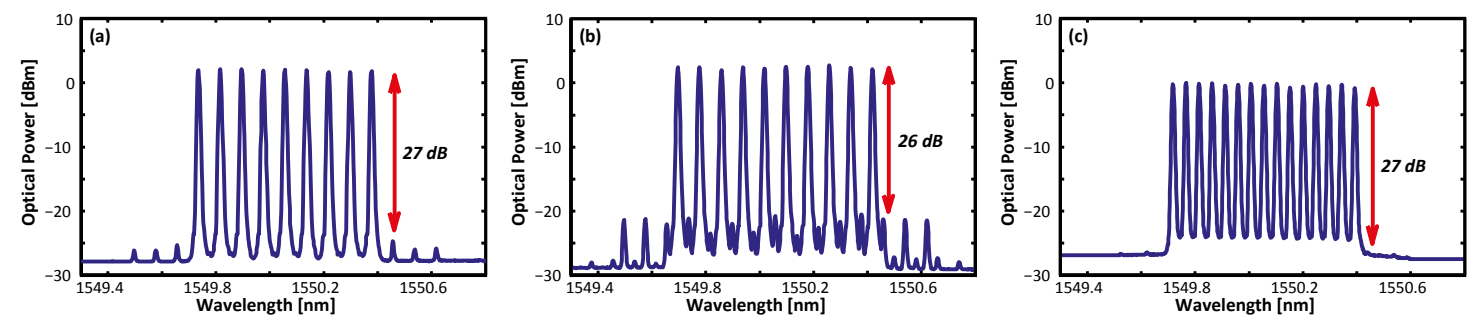

Fig. 3. Higly tunable generation of almost-ideal rectangular-shaped frequency combs using two cascaded intensity modulators. (a) Frequency comb with $N=9$ spectral lines, spectral separation $\Delta f=10 \mathrm{GHz}$ and bandwidth of $90 \mathrm{GHz}$. (b) Frequency comb with $N=10$, $\Delta f=10 \mathrm{GHz}$ and bandwidth of $100 \mathrm{GHz}$. (c) Frequency comb with $N=15, \Delta f=6 \mathrm{GHz}$ and bandwidth of $90 \mathrm{GHz}$. 
electrical domain, as shown in Fig. 2; this way, 5 spectral lines, corresponding to four first-order sidebands and carrier, can be obtained from each line generated by the first EOM. Thus, using the first modulator in carriersuppressed mode, a frequency comb with $N=10$ spectral lines has been produced. In particular, driving the first EOM at $25 \mathrm{GHz}$, and combining two RF signals at $10 \mathrm{GHz}$ and $20 \mathrm{GHz}$, respectively, to drive the second EOM, a comb with $\Delta f=10 \mathrm{GHz}$ and $100 \mathrm{GHz}$ bandwidth has been obtained, as reported in Fig. 3(b). By simply equalizing the power of the carrier with the first-order sidebands (generated at $30 \mathrm{GHz}$ ) in the first EOM according to Eq. (3), and by changing the frequency of both RF signals driving the second EOM to $6 \mathrm{GHz}$ and $12 \mathrm{GHz}$, a comb with $N=15, \Delta f=6 \mathrm{GHz}$, and bandwidth of $90 \mathrm{GHz}$ has been generated, as shown in Fig. 3(c). It is possible to observe that all frequency combs show a flat spectrum (with a maximum power variation among components of about $0.2 \mathrm{~dB}$ ) and a high suppression of out-of-band components (more than $26 \mathrm{~dB}$ suppression in all cases).
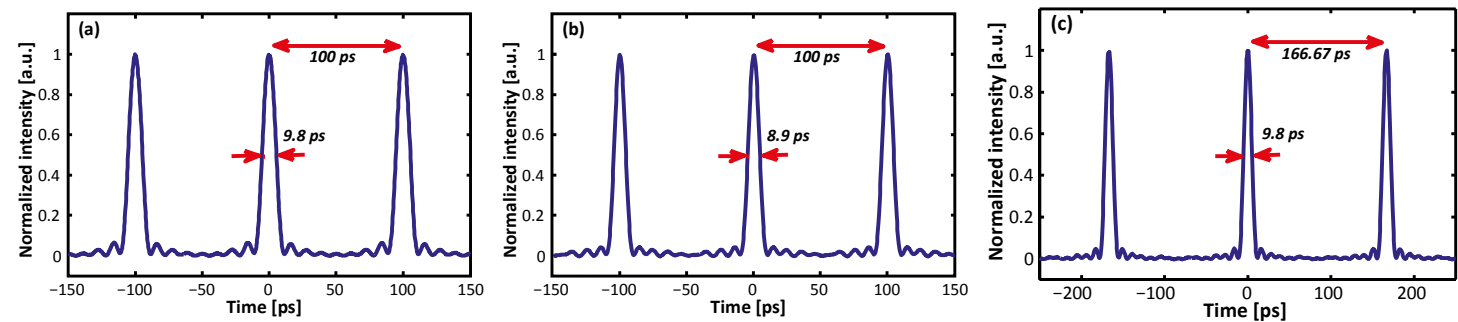

Fig. 4. Higly tunable generation of almost-ideal sinc-shaped Nyquist pulses resulting from the frequency combs shown in Fig. 3. (a) Sinc pulses with 9.8 ps FWHM duration and repetition period of 100 ps. (b) Sinc pulses with 8.9 ps FWHM duration and repetition period of 100 ps. (c) Sinc pulses with 9.8 ps FWHM duration and repetition period of 166.67 ps.

The time-domain measurements corresponding to the spectra reported in Fig. 3 are shown in Fig. 4. In particular, Fig. 4(a) shows a train of sinc pulses obtained from the frequency comb shown in Fig. 3(a). The full-width at halfmaximum (FWHM) of $9.8 \mathrm{ps}$ and the period of $T=100 \mathrm{ps}$ are given by the full bandwidth of $90 \mathrm{GHz}$ and the component spectral separation $\Delta f=10 \mathrm{GHz}$, respectively. Fig. 4(b) shows the sinc-shaped Nyquist pulses resulting from the spectrum reported in Fig 3(b). In this case, the use of an additional frequency component, leading to a bandwidth of $100 \mathrm{GHz}$, has reduced the sinc pulse duration down to $8.9 \mathrm{ps}$ (FWHM), maintaining the period of $100 \mathrm{ps}$, in accordance to the unchanged frequency spacing $\Delta f=10 \mathrm{GHz}$. Finally, Fig. 4(c) shows how the repetition period changed up to $166.67 \mathrm{ps}$ due to the reduced frequency spacing $\Delta f=6 \mathrm{GHz}$ reported in Fig. 3(c); the FWHM pulse duration has been maintained in 9.8 ps as in the first case.

The quality and stability of the generated sinc-shaped Nyquist pulses have been evaluated in terms of jitter and SNR. Fig. 5 shows a color-grade plot for pulses with 9.8 ps (FWHM) and repetition period of 100 ps, indicating an rms jitter of 82 fs (equivalent to $0.82 \%$ of the FWHM) and more than $40 \mathrm{~dB}$ SNR. A power difference below $1 \%$ between theoretical and experimental pulses has been verified by comparing measured pulses with the pulse intensity obtained from the field described in Eq. (1).

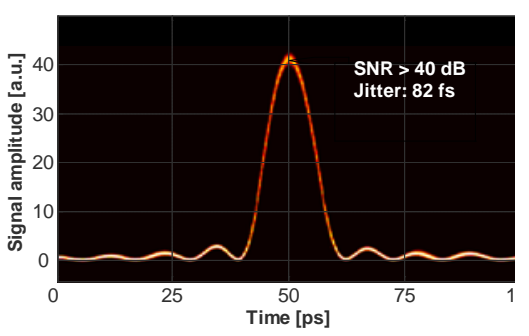

Fig. 5. Color-grade figure of the generated 9.8 ps (FWHM) sinc-shaped Nyquist pulses and repetition period of $100 \mathrm{ps}$.

\section{Conclusion}

In conclusion, a method to generate close-to-perfect, low-noise and highly-stable sinc pulses has been proposed using cascaded intensity modulators with a proper bias and modulating voltage adjustment. Experimental results demonstrate the high flexibility of the method to modify the pulse parameters thanks to the possibility to easily change the bandwidth of the frequency comb, the number of spectral components, and the frequency separation among them. Generated sinc-shaped Nyquist pulses can be straightforwardly combined with higher-order modulation formats to increase data transmission rates in telecommunications systems.

\section{References}

[1] R. Essiambre et al., “Capacity limits of optical fiber networks,” J. Lightwave Technol. 28, 662-701 (2010).

[2] G. Bosco et al., "Performance limits of Nyquist-WDM and CO-OFDM in high-speed PM-QPSK systems,” PTL 22 (15), 1129-1131 (2010).

[3] A. Wiberg et al., "Cavity-less pulse source based optical sampled ADC," in European Conference and Exhibition on Optical Communication ECOC 2012, OSA Technical Digest (online) (Optical Society of America, 2012), paper Mo.2.A.3.

[4] A. Vedadi et al., "Near-Nyquist optical pulse generation with fiber optical parametric amplification," Opt. Express 20, B558-B565 (2012).

[5] M. Nakazawa et al., "Ultrahigh-speed "orthogonal” TDM transmission with an optical Nyquist pulse train,” Opt. Express 20, 1129-1140 (2012).

[6] T. S. Sadeev et al., "Investigation and analysis of electro-optical devices in implementation of microwave photonic filters," in Proc. SPIE

8410, Optical Technologies for Telecommunications 2011 (January 20, 2012), paper 841007. 